\title{
PRESENTATION OF SET FUNCTORS: A COALGEBRAIC PERSPECTIVE
}

\author{
J. ADÁMEK, H. P. GUMM, V. TRNKOVÁ
}

\begin{abstract}
Accessible set functors can be presented by signatures and equations as quotients of polynomial functors. We determine how preservation of pullbacks and other related properties (often applied in coalgebra) are reflected in the structure of the system of equations.
\end{abstract}

\section{INTRODUCTION}

With the recent development of General Coalgebra as a general theory of state based systems [13], the study of properties of set functors has seen a renewed rise of interest. The reason is that every set functor models its own type of coalgebras. Systems as varied as streams, automata (with alphabet $\Sigma$ and output set $D$ ), Kripke structures, probabilistic transition systems or topological spaces can be modeled as coalgebras, when we use as type functor the identity functor $I$, a polynomial functor $D \times(-)^{\Sigma}$, the powerset functor $\mathbb{P}$, the finite distribution functor $\mathcal{D}$ or the filter functor $\mathbb{F}$, respectively.

Not surprisingly, the properties of the type functor influence strongly the coalgebraic properties of the corresponding structures. For instance, the type functor $F$ preserves weak kernel pairs iff each congruence relation is a bisimulation [8], it preserves inverse images iff the category $\operatorname{CoAlg}(F)$ of all $F$-coalgebras is extensive, see [6]. Preservation of inverse images is also relevant for recursive coalgebras, see [15], and $F$ preserves arbitrary intersections iff each coalgebra is a union of onegenerated subcoalgebras [14], etc. Therefore it often becomes necessary to decide whether a given set functor has one of the mentioned properties.

Fortunately, a large body of knowledge about set functors was accumulated in the 1970 's, see for example $[11,16,17,18]$. Our main tool is the presentation of every set functor as a class-directed union of accessible functors, and the presentation of every accessible functor as a quotient of a polynomial one. The latter means that for every accessible functor we have an equational presentation (using flat terms). For example, the finite-powerset functor is described by one $n$-ary operation $\sigma_{n}$ for every $n \in \mathbb{N}$ and by the equations $\sigma_{n}(x)=\sigma_{m}(y)$ whenever $x$ and $y$ have the same image.

The aim of the present paper is to express preservation properties of set functors, often encountered in general coalgebra, in the language of equational presentations. For example, an accessible functor preserves weak pullbacks iff its presentation is dominating; this means that all equations are generated (in a sense made precise

Date: April8, 2008.

The third author gratefully acknowledges the support of MSM 0021620839, a project of the Czech Ministry of Education and of the grant 201/06/0664 by the Grant Agency of Czech Republic. 
below) by equations of the form $\sigma\left(i d_{n}\right)=\tau(y)$ for $n$-ary symbols $\sigma$. It follows that $\mathbb{P}_{\omega}$ weakly preserves pullbacks but almost none of its subfunctors $\mathbb{P}_{n}$ does.

For wide pullbacks we extend a result of A. Joyal [10] from finitary to accessible functors: we prove that an accessible functor weakly preserves wide pullbacks iff it is analytic.

\section{Preliminaries}

2.1. Pullbacks, inverse images and intersections. Recall that a pullback of a cocone $f_{i}: A_{i} \rightarrow A, i=1,2$, is a limit of that cocone, that is, a commutative square

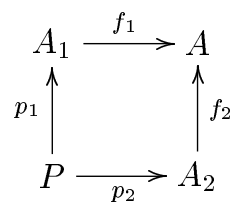

with the universal property that for every commutative square $f_{1} \circ q_{1}=f_{2} \circ q_{2}$ there exists a unique mediating morphism $d$, i.e. a morphism for which the diagram

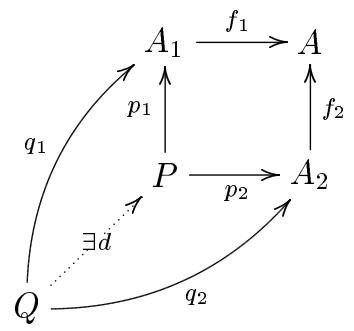

commutes. If the pullback $\left(P, p_{1}, p_{2}\right)$ of $f_{1}$ and $f_{2}$ exists, then it is unique up to isomorphism. If $f_{1}$ is monic, then $p_{2}$ is monic and the pullback is called the inverse image of the subobject $f_{1}: A_{1} \rightarrow A$ under $f_{2}: A_{2} \rightarrow A$. If both $f_{1}$ and $f_{2}$ are monic, the pullback is known as a (finite) intersection.

In the category Set of sets and mappings, in which we shall exclusively work in this paper, the pullback of $f_{1}$ and $f_{2}$ always exists. It is given by

$$
P:=\left\{\left(a_{1}, a_{2}\right) \in A_{1} \times A_{2} \mid f_{1}\left(a_{1}\right)=f_{2}\left(a_{2}\right)\right\}
$$

and $p_{1}$ and $p_{2}$ are the domain restrictions of the canonical projections $\pi_{i}\left(a_{1}, a_{2}\right)=a_{i}$ for $i=1,2$.

When $f_{2}$ is a subset inclusion $\iota: A_{2} \hookrightarrow A$, the pullback simplifies to $\left\{a_{1} \mid\right.$ $\left.f_{1}\left(a_{1}\right) \in A_{2}\right\}=f_{1}^{-1}\left[A_{2}\right]$ with $p_{1}$ the natural inclusion of $f_{1}^{-1}\left[A_{2}\right]$ in $A_{1}$ and $p_{2}=$ $f_{1}^{\prime}: f_{1}^{-1}\left[A_{2}\right] \rightarrow A_{2}$, the domain-codomain restriction of $f_{1}$.

Specializing further, when $f_{1}: A_{1} \hookrightarrow A$ and $f_{2}: A_{2} \hookrightarrow A$ are both subset inclusions, then their pullback is given by the intersection $A_{1} \cap A_{2}$ with $p_{1}$ and $p_{2}$ the inclusions of $A_{1} \cap A_{2}$ in $A_{1}$ and in $A_{2}$. 
Generalizing the above notion of (binary) pullback, a wide pullback of a family of morphisms $\left(f_{i}: A_{i} \rightarrow A\right)_{i \in I}$ is the limit $\left(p_{i}: P \rightarrow A_{i}\right)_{i \in I}$ of this sink:

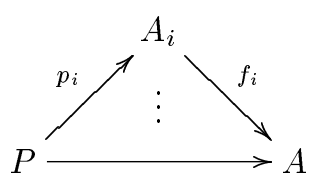

If all $f_{i}$ are monic, then the wide pullback is called a (wide) intersection of the subobjects $\left(f_{i}\right)_{i \in I}$.

2.2. Weak pullbacks. If in the above definition of the pullback of $f_{1}: A_{1} \rightarrow A$ and $f_{2}: A_{2} \rightarrow A$ we drop the uniqueness condition for the mediating morphism $d$, we obtain the notion of weak pullback.

Note that, if $\left(P, p_{1}, p_{2}\right)$ is a weak pullback and one of $p_{1}$ or $p_{2}$ is monic, then it is already a (real) pullback. More generally, a weak pullback square (2.1) is a pullback square iff the morphisms $p_{i}$ are jointly monic.

Pullbacks and weak pullbacks can be combined, and under certain conditions be split apart, as is stated in the following lemma from [12] whose proof is an easy exercise:

Lemma 2.1. Let in the following diagram all squares be commutative, then

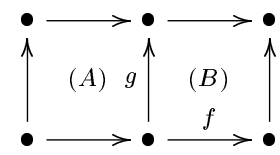

(i) if $(A)$ and $(B)$ are (weak) pullback squares, then so is the perimeter $(A, B)$

(ii) if the perimeter $(\mathrm{A}, \mathrm{B})$ is a (weak) pullback square, and if $f, g$ are jointly monic, then $(A)$ is a (weak) pullback square.

Since functors preserve composition o of morphisms, and identities $i d_{X}$, they preserve every property that can be positively expressed in the language of $\circ$ and $i d$. In particular they preserve commutativity of diagrams.

In the category Set, each epimorphism $f$ is right-invertible, i.e. $\exists \bar{f} . f \circ \bar{f}=i d$ and each monomorphism $g$ with nonempty domain is left-invertible, i.e. $\exists \bar{g} . \bar{g} \circ g=i d$. Consequently, set functors $F$ preserve this property, so if $f: X \rightarrow Y$ is epi, then $F f: F X \rightarrow F Y$ is epi, too, and if $X \neq \emptyset$ and $f: X \rightarrow Y$ is monic, then so is Ff : $F X \rightarrow F Y$. In short: each set functor preserves epics, and all monics with the possible exception of the empty maps $\emptyset_{X}: \emptyset \rightarrow X$ for any set $X$.

2.3. Preservation of intersections. Given a pullback (2.1), then a commutative square (2.2) is a weak pullback iff the mediating morphism $d$ is a split epimorphism. Therefore, a functor weakly preserves pullbacks (i.e. maps pullback squares to weak pullback squares) iff $F$ preserves weak pullbacks. Analogously for other limits, such as weak wide pullbacks.

In [8] it is shown that a functor weakly preserves (finite) pullbacks iff it weakly preserves inverse images and kernel pairs. Nonempty intersections, as has been shown in [16], are preserved for free:

Proposition 2.2. ([16]) Every set functor preserves finite nonempty intersections. 
To see this, consider the commutative diagram representing a nonempty intersection in the category Set, where the hooked arrows denote the inclusion maps.

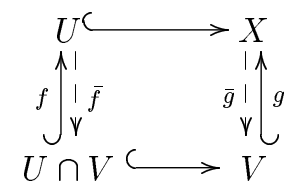

When $U \cap V$ is not empty, we can find left inverses $\bar{f}$ and $\bar{g}$ to the inclusion maps $f$ and $g$, and with just a little care, we can choose them so that the inner diagram, delineated by $\bar{f}$ and $\bar{g}$, is commutative, too. Conversely, given a commutative diagram as above, where the inner square commutes, too, and where $g \circ \bar{g} \circ g=g$, it is easy to check that the diagram must be a pullback, i.e. an intersection. Thus, being an intersection has been coded as a positive statement in the language of 0 and $i d$ and must therefore be preserved by any set functor $F$ (see [7]).

The restriction to nonempty intersections in the above proposition is, at first sight, indispensable. Consider, for instance, any functor $F$ with $F \emptyset \neq \emptyset$. One obtains a different functor $F^{\prime}$ by setting $F^{\prime} \emptyset:=\emptyset$ and $F^{\prime} X=F X$ otherwise. Clearly, either $F$ or $F^{\prime}$ must fail to preserve empty intersections. Fortunately, as we shall see, any functor can be "repaired" by just modifying it on the empty set and on mappings with empty domain so that the resulting functor $F^{\prime}$ preserves all finite intersections and all monos.

2.4. Soundness. Let $C_{1}$ be the constant functor with value 1 and $C_{1,0}$ the functor which agrees with $C_{1}$ on all nonempty sets and mappings, except that $C_{1,0} \emptyset=\emptyset$.

Definition 2.3. A set functor is called sound if every natural transformation $C_{1,0} \rightarrow F$ has a unique extension to $C_{1} \rightarrow F$.

Fact 2.4. Every set functor $F$ can be modified at $\emptyset$ to obtain a sound functor $F^{\prime}$. That is

- $F^{\prime}$ agrees with $F$ on all nonempty sets and on all mappings with nonempty domain

- $F^{\prime}$ is sound.

One way to obtain $F^{\prime}$ is by replacing $F \emptyset$ by the set of all natural transformations nat $\left(C_{1,0}, F\right)$ where $C_{1,0}$ is the functor with object map

$$
C_{1,0}(X)=\left\{\begin{array}{ll}
\emptyset & \text { if } X=\emptyset \\
1 & \text { else }
\end{array} .\right.
$$

Notice that every element in $F^{\prime}(\emptyset):=\operatorname{nat}\left(C_{1,0}, F\right)$ corresponds to a collection of elements $\delta_{X} \in F X$ for each set $X$ such that $F f\left(\delta_{X}\right)=\delta_{Y}$ whenever $f: X \rightarrow Y$. Therefore, the elements of $F^{\prime}(\emptyset)$ are called distinguished points. On the empty maps $\emptyset_{X}$, the functor $F^{\prime}$ is defined as

$$
\left(F^{\prime} \emptyset_{X}\right)(\delta):=\delta_{X}
$$

for each distinguished point $\delta \in F^{\prime} \emptyset$. It is now easy to prove that $F^{\prime}$ preserves all finite intersections.

Proposition 2.5. [17] Every sound functor preserves monomorphisms and finite intersections. 
Example 2.6. The functors $\mathbb{P}, \mathbb{P}_{\omega}$ and $(-)^{n}$ are sound. The sound modification of $C_{1,0}$ is $C_{1}$.

2.5. Sound subfunctors. Recall that a natural transformation $\nu$ between two functors $F$ and $G$ is called cartesian, if each naturality square is a pullback. We shall call a transformation $\nu: F \rightarrow G$ sub-cartesian, if for every injective map $f: X \hookrightarrow Y$ the naturality square $G f \circ \nu_{X}=\nu_{Y} \circ F f$ is a pullback square. Related to Proposition 2.2 is the following result from T. Schröder's thesis [14]. We shall supply the proof here, since in the original [14], Thm. 4.15, the case where $X$ is empty was not treated correctly.

Proposition 2.7. Any injective natural transformation between sound set functors is sub-cartesian.

Proof. Consider an injective map $f: X \hookrightarrow Y$ and an injective natural transformation $\nu: F \hookrightarrow G$. Assume first $X \neq \emptyset$, then we can find a left inverse $\bar{f}: Y \rightarrow X$, so that $\bar{f} \circ f=i d_{X}$. Consider the naturality diagram below.

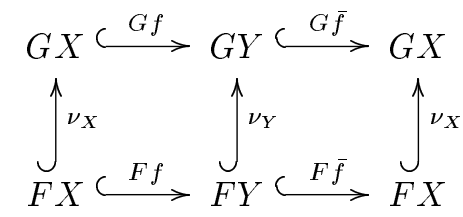

Since $F \bar{f} \circ F f=i d_{F X}$ and $G \bar{f} \circ G f=i d_{G X}$, the perimeter square is a pullback. By Lemma 2.1, the left square must be a pullback, too. The case $X=Y=\emptyset$ is trivial, since $F f=F_{i d_{\emptyset}}=i d_{F \emptyset}$ and likewise $G f=i d_{G \emptyset}$ are isomorphisms. Finally, assume $X=\emptyset$ and $Y \neq \emptyset$. Consider the following cube, in which the bottom square is a pullback and $G \emptyset_{Y}: G \emptyset \rightarrow G Y$ is monic as a result of $F$ and $G$ being sound. The back face is a pullback by the previous case. A diagram chase reveals that the front face must be a pullback, too, finishing the proof.

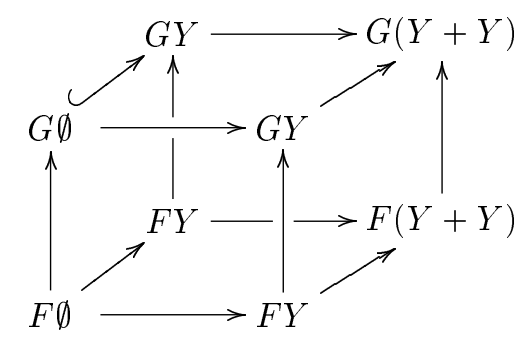

2.6. Decomposition of Set Functors. For every set functor $F$ we have:

(i) if $F 1=\emptyset$ then $F$ is the constant functor $C_{\emptyset}$ with value $\emptyset$,

(ii) if $F 1 \cong 1$ then $F$ is indecomposable, that is, $F=G+H$ implies $G=C_{\emptyset}$ or $H=C_{\emptyset}$, and

(iii) in general, $F$ is a coproduct of indecomposable functors

$$
F=\coprod_{i \in F 1} F_{i}
$$

where $F_{i}$, for each $i \in F 1$, is the subfunctor of $F$ given as the inverse image of $\{i\}$ under $F !_{X}$, where $!_{X}$ is the trivial map $!_{X}: X \rightarrow 1$. 
Indeed, the first claim follows from applying $F$ to $!_{X}: X \rightarrow 1$, the next is an easy consequence, and the fact that the $F_{i}$ are subfunctors of $F$ follows from the fact that $!_{X}=!_{Y} \circ f$ for any $f: X \rightarrow Y$. The functors $F_{i}$ are called the components of $F$.

Example 2.8. The powerset functor $\mathbb{P}$ has two components: $C_{1}$, the constant functor sending every set $X$ to $1=\{\emptyset\}$, and its complement, the nonempty-subsets functor.

\section{Accessible Set Functors}

A set functor $F$ is called accessible if it preserves, for some infinite cardinal $\lambda$, all $\lambda$-filtered colimits. Given such a $\lambda$, we call $F \lambda$-accessible. Recall that a category is $\lambda$-filtered iff for every subcategory of less than $\lambda$ objects it contains a cocone; colimits of diagrams indexed by small, $\lambda$-filtered categories are called $\lambda$-filtered colimits. Since every set $X$ is a $\lambda$-filtered union of its subsets of cardinality less than $\lambda$, this implies the following representation of $F$ (we use $\iota: U \hookrightarrow X$ to denote set inclusion):

$$
F X=\bigcup_{U \subseteq X} \bigcup_{|U|<\lambda} F \iota[F U]
$$

In fact, having such a representation is equivalent for $F$ to be $\lambda$-accessible, see [3].

Obviously, products, coproducts, and subfunctors of accessible functors are all accessible. The $\omega$-accessible functors are called finitary.

Given an arbitrary functor $F$ and any cardinal $\lambda$, we can define a subfunctor $F_{\lambda}$ of $F$ by

$$
F_{\lambda} X:=\bigcup_{n<\lambda} \bigcup_{f \in X^{n}} F f[F n] .
$$

Then $F$ is $\lambda$-accessible iff $F=F_{\lambda}$. Clearly, we have for any functor $F$ that

$$
F=\bigcup_{\lambda \in \text { Card }} F_{\lambda}
$$

provided that we allow unions indexed over a proper class.

In coalgebra, a somewhat different condition is often used: $F$ is called bounded if for some infinite cardinal $\lambda$ every element of a coalgebra $A$ lies in a subcoalgebra of cardinality at most $\lambda$. Equivalently, (see [7]) every coalgebra has a subcoalgebra of size at most $\lambda$. Accessible functors are precisely the bounded ones, as proved in $[3,7]$.

Example 3.1. The powerset functor $\mathbb{P}$ is an example of a non-accessible functor. The finite-powerset functor $\mathbb{P}_{\omega}$ is a finitary subfunctor of $\mathbb{P}$. The countablepowerset functor $\mathbb{P}_{\omega_{1}}$, assigning to every set the set of all its countable subsets, is $\omega_{1}$-accessible.

\subsection{Signatures and polynomial functors. We denote by}

$$
Q_{n}(n \text { a cardinal })
$$

the functor of the $n$-th cartesian power, $X \mapsto X^{n}$. If we consider $n$ as the set of all smaller ordinals, then $Q_{n}$ is obviously naturally isomorphic to hom $(n,-)$. The functor $Q_{n}$ is accessible: choose $\lambda$ to be the first infinite cardinal with $\lambda>n$.

Sums of the cartesian power functors are called polynomial functors: 


$$
H_{\Sigma} X=\coprod_{\sigma \in \Sigma} X^{a r(\sigma)} .
$$

Here, the indexing set $\Sigma$ is a signature, that is a collection of symbols $\sigma$, each of which is equipped with a cardinal $\operatorname{ar}(\sigma)$, called the arity of $\sigma$. We define $\Sigma_{n}:=$ $\{\sigma \in \Sigma \mid \operatorname{arity}(\sigma)=n\}$.

Every element of $H_{\Sigma} X$ is uniquely identified by its component $\sigma \in \Sigma$ and by a map $x: \operatorname{ar}(\sigma) \rightarrow X$. We use the notation

$$
\sigma(x)
$$

rather than $(\sigma, x)$ for the element $x$ in the component $\sigma$. We call the expressions $\sigma(x)$ flat terms. When $\operatorname{ar}(\sigma)=n$ is finite, then $x: n \rightarrow X$ is sometimes represented by the tuple $\left(x_{0}, \ldots, x_{n-1}\right)$, where $x_{i}=x(i)$ and consequently, the flat term $\sigma(x)$ is written as $\sigma\left(x_{0}, \ldots, x_{n-1}\right)$. The action of $H_{\Sigma}$ on a function $u: X \rightarrow Y$ is

$$
H_{\Sigma} u=\sigma(x) \mapsto \sigma(u \circ x)
$$

for all $\sigma \in \Sigma_{n}$, and all $x: n \rightarrow X$.

Example 3.2. The components of $H_{\Sigma}$ are the individual (indecomposable) functors $Q_{n}$.

3.2. Quotients and congruences. A quotient of a functor $F$ is the usual concept applied to the category of all set functors and natural transformations: a quotient of $F$ is represented by an epimorphism, that is a natural transformation $\varepsilon: F \rightarrow G$ whose components $\varepsilon_{X}: F X \rightarrow G X$ are epimorphisms in Set for each object $X$.

A quotient of a $\lambda$-accessible functor is $\lambda$-accessible. In fact, let (3.1) hold and let $x$ be an element of $G X$. Choose $x^{\prime} \in F X$ with $x=\varepsilon_{X}\left(x^{\prime}\right)$ and use (3.1) to find $f: n \rightarrow X$ and $y^{\prime} \in F n$ with $F f\left(y^{\prime}\right)=x^{\prime}$. Then for $y=\varepsilon_{Y}\left(y^{\prime}\right)$ we have $x=\varepsilon_{X}\left(F f\left(y^{\prime}\right)\right)=G f\left(\varepsilon_{Y}\left(y^{\prime}\right)=G f(y)\right.$.

Every quotient of $F$ defines a congruence, that is a collection of equivalence relations $\sim_{X}$ on $F X$ for all sets $X$ such that for every morphism $f: X \rightarrow Y$ we have: if $x \sim_{X} x^{\prime}$ then $F f(x) \sim_{Y} F f\left(x^{\prime}\right)$. Given a quotient $\tau: F \rightarrow Q$, then the kernels of the $\tau_{X}$ form a congruence, and conversely, each congruence $\sim$ defines a quotient with $G X=F X / \sim_{X}$ where $\varepsilon_{X}: F X \rightarrow G X$ is the canonical map sending $x \in F X$ to its equivalence class $[x] \sim_{X}$.

Congruences on a functor $F$ are partially ordered in the usual sense: if $\sim$ and $\approx$ are two congruences, then $\sim$ is less than $\approx$ if for every set $X$ the relation $\sim_{X}$ on $F X$ is contained in $\approx_{X}$.

Example 3.3. $\quad$ (i) Let $F$ be the functor of all unordered pairs:

$$
F X=\left\{\left\{x_{1}, x_{2}\right\} \mid x_{i} \in X \text { for } i=1,2\right\}
$$

This is a quotient of $Q_{2}$ modulo the least congruence with

$$
(x, y) \sim_{X}(y, x) \text { for all } x, y \in X .
$$

(ii) Another quotient of $Q_{2}$ is obtained by merging the diagonal into a single element (called $d$ ):

$$
D X=\left(X \times X-\Delta_{X}\right)+\{d\} .
$$


For morphisms $f: X \rightarrow Y$ we put $(D f)(d)=d$ and

$$
D f\left(x, x^{\prime}\right)= \begin{cases}\left(f(x), f\left(x^{\prime}\right)\right) & \text { if } f(x) \neq f\left(x^{\prime}\right) \\ d & \text { otherwise. }\end{cases}
$$

$D$ is the quotient of $Q_{2}$ modulo the least congruence with

$$
(x, x) \sim_{X}(y, y) \text { for all } x, y \in X .
$$

(iii) $\mathbb{P}_{\omega_{1}}$ is the quotient of $C_{1}+Q_{\omega}$ obtained by identifying $x, y: \omega \rightarrow X$ iff their images agree, i.e. if $x[\omega]=y[\omega]$.

(iv) $\mathbb{P}_{\omega}$ is the quotient of $H_{\Sigma}$ with $\Sigma=\left(\sigma_{n}\right)_{n \in \mathbb{N}}$ and $\operatorname{ar}\left(\sigma_{n}\right)=n$ for all $n \in \mathbb{N}$, obtained by identifying $\sigma_{m}(x)$ with $\sigma_{n}(y)$ iff $x[m]=y[n]$.

3.3. Equational presentations. Taking quotients of polynomial functors leads to identifications of flat terms. Borrowing notations and conventions from algebraic logic, we call a triple $(X, \sigma(x), \tau(y))$ where $X$ is a set and $\sigma(x)$ and $\tau(y) \Sigma$-terms in $H_{\Sigma} X$ an equation and write it: $\sigma(x) \overline{\bar{X}} \tau(y)$.

Definition 3.4. An equational presentation of a set functor $F$ is a signature $\Sigma$ together with a set $E$ of equations such that $F$ is the quotient $H_{\Sigma} / \sim$ of the polynomial functor of $\Sigma$ modulo the least congruence $\sim$ such that $\sigma(x) \sim_{X} \tau(y)$ holds for every equation $\sigma(x) \underset{\bar{X}}{\tau} \tau(y)$ in $E$.

The following example demonstrates that we need to clarify the logic of equations we use for set functors.

Example 3.5. The functor $C_{1,2}$ given by $\emptyset \mapsto 2$ and $X \mapsto 1$ for $X \neq \emptyset$ has an equational presentation in the signature $\Sigma=\left\{c_{1}, c_{2}, \sigma\right\}$ with $c_{1}, c_{2}$ nullary and $\sigma$ unary, given by the equations

$$
\sigma(x)=c_{1} \text { and } \sigma(x)=c_{2} .
$$

However, the equation $c_{1}=c_{2}$ does not hold in the presentation of $C_{1,2}$.

Fortunately, this example is less disquieting than it might seem at first sight: the functor $C_{1,2}$ is not sound (see 2.3) and we will show that our Equational Logic is adequate for all sound set functors.

Lemma 3.6. For every presentation $(\Sigma, E)$ of a sound functor the valid equations $\sigma(x) \overline{\bar{X}} \tau(y)$ are independent of the set $X$ of variables in the following sense: if $X^{\prime}$ is the set of variables that appear in $\sigma(x)$ or $\tau(y)$ then $E=\sigma(x) \overline{\bar{X}} \tau(y)$ iff $E=\sigma(x) \underset{\overline{X^{\prime}}}{\tau} \tau(y)$.

Proof. Let $F$ be the sound functor presented by $E$ and let $\sim$ be the corresponding congruence. Given a commutative diagram

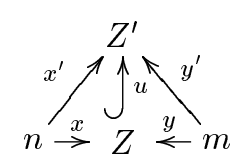

where $u: Z \hookrightarrow Z^{\prime}$ is the subset inclusion, then for every $\sigma \in \Sigma_{n}$ and $\tau \in \Sigma_{m}$ we have

$$
\sigma(x) \sim_{Z} \tau(y) \text { iff } \sigma\left(x^{\prime}\right) \sim_{Z^{\prime}} \tau\left(y^{\prime}\right) .
$$


This follows from (3.3) and the fact that $F u$ is a monomorphism, see Proposition 2.5 , since

$$
\varepsilon_{Z^{\prime}}\left(\sigma\left(x^{\prime}\right)\right)=\varepsilon_{Z^{\prime}}(\sigma(u \circ x))=\left(\varepsilon_{Z^{\prime}} \circ H_{\Sigma} u\right)(\sigma(x))=F u\left(\varepsilon_{Z}(\sigma(x))\right)
$$

and analogously for $\tau\left(y^{\prime}\right)$. Consequently, $\varepsilon_{Z}$ merges $\sigma(x)$ and $\tau(y)$ iff $\varepsilon_{Z^{\prime}}$ merges $\sigma\left(x^{\prime}\right)$ and $\tau\left(y^{\prime}\right)$.

Notation 3.7. Due to Lemma 3.6 we need not mention the variable set $X$ when writing equations for sound functors.

\section{Example 3.8.}

(i) The unordered-pairs functor, see Example 3.3(i), is presented by a binary operation symbol $\sigma$ and the equation

$$
\sigma\left(x_{0}, x_{1}\right)=\sigma\left(x_{1}, x_{0}\right)
$$

(ii) The functor $D$ of Example 3.3(ii) is presented by

$$
\sigma\left(x_{0}, x_{0}\right)=\sigma\left(x_{1}, x_{1}\right) .
$$

(iii) The finite-powerset functor $\mathbb{P}_{\omega}$ can be presented by a signature $\Sigma$ having precisely one $n$-ary operation $\sigma_{n}$ for every natural number $n$ :

$$
H_{\Sigma}(X)=1+X+X^{2}+\cdots
$$

modulo the equations

$$
\sigma_{n}\left(x_{0}, \ldots, x_{n-1}\right)=\sigma_{m}\left(y_{0}, \ldots, y_{m-1}\right)
$$

for all pairs $m, n$ of natural numbers and all tuples with $\left\{x_{0}, \ldots, x_{n-1}\right\}=$ $\left\{y_{0}, \ldots, y_{m-1}\right\}$.

(iv) The countable-powerset functor $\mathbb{P}_{\omega_{1}}$ (see example 3.1), has a simple presentation: let $\Sigma=\{c, \sigma\}$ where $c$ is nullary and $\sigma$ is an $\omega$-ary operation symbol. $E$ is the set of all equations

$$
\sigma\left(x_{n}\right)_{n<\omega}=\sigma\left(y_{n}\right)_{n<\omega}
$$

with $\left\{x_{n} \mid n \in \omega\right\}=\left\{y_{n} \mid n \in \omega\right\}$ for $x, y: \omega \rightarrow \omega$.

Proposition 3.9. Every accessible functor has an equational presentation.

Remark. If $F$ is $\lambda$-accessible, we prove that it has a $\lambda$-ary presentation as follows: Let $\Sigma$ be the $\lambda$-ary signature whose $n$-ary symbols are labeled by the elements of $F(n)$ :

$$
\Sigma_{n}=F(n) \text { for all cardinals } n<\lambda .
$$

Let $E$ be the set of all equations $\sigma(x)=\tau(y)$, where $\sigma \in F(n)$ and $\tau \in F(m)$, and for $k=\max (n, m)$ we have

$$
x: n \rightarrow k \text { and } y: m \rightarrow k \text { with } F x(\sigma)=F y(\tau) .
$$

Proof. To show that the above is an equational presentation of $F$ define a natural transformation

to have components

$$
\varepsilon: H_{\Sigma} \rightarrow F
$$

$$
\varepsilon_{X}: \coprod_{n<\lambda} F(n) \times X^{n} \rightarrow F X
$$

given by the Yoneda transformations

$$
(\sigma, x) \mapsto F x(\sigma) \text { for } \sigma \in F(n) \text { and } x: n \rightarrow X .
$$


This is an epi-transformation whose kernel defines the above presentation

$$
E=\bigcup_{n<\lambda} \operatorname{ker} \varepsilon_{n}
$$

of the functor $F$.

Remark 3.10. Non-accessible set functors do not have an equational presentation. (We would need to admit signatures $\Sigma$ having a proper class of symbols, which would render $H_{\Sigma} 1$ a proper class.) However, every set functor can be represented as class-directed unions of accessible functors. Recall, that a $\lambda$-directed union is such that any $n$-tuple of its members with $n<\lambda$ is contained in a single member. A class-directed union is a union which is $\lambda$-directed for every cardinal $\lambda$. For every set functor $F$ the union of the subfunctors in Formula (3.2)

$$
F=\bigcup_{\lambda \in \text { Card }} F_{\lambda}
$$

is class directed.

3.4. Equational Logic. An equational presentation $(\Sigma, E)$ may entail further equations, originally not present in $E$, but contained in the congruence relation on $H_{\Sigma}$ generated by $E$. These equations can be derived by a set of rules reminiscent of the deduction rules of Birkhoff's equational logic. But, since we are dealing with flat terms, these rules have a particularly simple form:

Definition 3.11. The equational deduction system for flat equations consists of one axiom

$$
\overline{\rho(z)=\rho(z)}
$$

and three deduction rules

$$
\begin{gathered}
\frac{\rho(z)=\rho^{\prime}\left(z^{\prime}\right)}{\rho^{\prime}\left(z^{\prime}\right)=\rho(z)} \\
\frac{\rho(z)=\rho^{\prime}\left(z^{\prime}\right), \rho^{\prime}\left(z^{\prime}\right)=\rho^{\prime \prime}\left(z^{\prime \prime}\right)}{\rho(z)=\rho^{\prime \prime}\left(z^{\prime \prime}\right)} \\
\frac{\rho(z)=\rho^{\prime}\left(z^{\prime}\right)}{\rho(u \circ z)=\rho^{\prime}\left(u \circ z^{\prime}\right)}
\end{gathered}
$$

where the last rule applies to all functions $u$ with $u \circ z$ and $u \circ z^{\prime}$ defined.

The above deduction rules introduce the usual notion of derivability. To be explicit, a formal proof of an equation $e \equiv \sigma(x)=\tau(y)$ is a sequence $e_{1}, e_{2}, \ldots, e_{n}$ of equations with $e_{n} \equiv e$, and where for each $i=1, \ldots, n$ either $e_{i}$ is an equation in $E$ or $e_{i}$ is the conclusion of one of the deduction rules whose assumptions lie in $\left\{e_{1}, e_{2}, \ldots, e_{i-1}\right\}$. We write $E \vdash e$, if there is a formal proof of $e$ from $E$.

Lemma 3.12. [Adequacy of Equational Logic] Let $F$ be a sound accessible functor. Then for every equational presentation $(\Sigma, E)$ of $F$ we have $F \cong H_{\Sigma} / \sim$ for the congruence $\sim$ of $H_{\Sigma}$ defined by

$$
\sigma(x) \sim \tau(y) \text { iff } E \vdash \sigma(x)=\tau(y) .
$$


Proof. We prove that the rules (3.4) to (3.7) define the least congruence on $H_{\Sigma}$ containing $E$. Then $F \cong H_{\Sigma} / \sim$ follows from the definition of equational presentations.

Due to lemma 3.6, the relation $\sim$ is well defined by equation (3.8). It is reflexive due to (3.4), symmetric due to (3.5) and transitive due to (3.6). The system $\sim$ of these equivalence relations is a congruence of $H_{\Sigma}$, that is, for every morphism $u: Z \rightarrow Z^{\prime}$ we have that ${ }^{1}$

$$
\sigma(x) \sim_{Z} \tau(y) \Longrightarrow \sigma(u \circ x) \sim_{Z^{\prime}} \tau(u \circ y),
$$

due to (3.7). By definition, $\sim$ contains $E$.

Let $\approx$ be a congruence on $H_{\Sigma}$ containing $E$, then $\approx$ contains $\sim$. In fact, given $\sigma(x) \sim_{Z} \tau(y)$, we prove $\sigma(x) \approx_{Z} \tau(y)$ by induction on the length $n$ of the proof $e_{1}, \ldots, e_{n}(\equiv \sigma(x)=\tau(y))$. If $n=1$ then either $\sigma(x)$ is the same term as $\tau(y)$, or the equation $\sigma(x)=\tau(y)$ lies in $E$; in both cases $\sigma(x) \approx_{Z} \tau(y)$.

In the induction step, we have $n>1$ and there are three cases corresponding to the three deduction rules applicable in the last step $e_{n}$ of the formal proof:

- case (3.5) means that there exists $i<n$ with $e_{i} \equiv(\tau(y)=\sigma(x))$ an equation in $H_{\Sigma} Z^{\prime}$ for some set $Z^{\prime}$ of variables. By Lemma 3.6, the set of variables plays no role, thus, by induction hypothesis we obtain $\tau(y) \approx_{Z^{\prime}}$ $\sigma(x)$ and this implies $\sigma(x) \approx_{Z^{\prime}} \tau(y)$,

- case (3.6) is analogous to (3.5), and

- case (3.7) means that there is $i<n$ with

$$
e_{i} \equiv\left(\rho(z)=\rho^{\prime}\left(z^{\prime}\right)\right)
$$

such that for some $u: Z^{\prime} \rightarrow Z$ we have

$$
\sigma(x) \equiv \rho(u \circ z) \text { and } \tau(y) \equiv \rho^{\prime}\left(u \circ z^{\prime}\right) .
$$

This implies $\sigma=\rho$ and $\tau=\rho^{\prime}$. By induction hypothesis (and since extra variables play no role) we have $\rho(z) \approx_{Z^{\prime}} \rho^{\prime}\left(z^{\prime}\right)$. As $\approx$ is a congruence of $H_{\Sigma}$, this implies

$$
\sigma(x) \equiv \rho(u \circ z) \approx_{Z} \rho^{\prime}\left(u \circ z^{\prime}\right) \equiv \tau(y) .
$$

\section{Preservation of Wide Intersections}

We have seen in Proposition 2.5 that set functors "essentially" preserve finite intersections. In the present section, we characterize set functors preserving wide intersections.

Definition 4.1. Given a flat equational presentation $(\Sigma, E)$, we call a term $\sigma\left(x_{i}\right)_{i<n}$ reduced, if for every equation

$$
E \vdash \sigma\left(x_{i}\right)_{i<n}=\tau\left(y_{j}\right)_{j<m}
$$

the variables on the left-hand side are contained in the variables of the right-hand:

$$
\left\{x_{i}\right\}_{i<n} \subseteq\left\{y_{j}\right\}_{j<m}
$$

We call the presentation reduced, provided that for every flat term $\rho\left(z_{k}\right)$ there exists a reduced term $\sigma\left(x_{i}\right)$ such that $E \vdash \rho\left(z_{k}\right)=\sigma\left(x_{i}\right)$.

\footnotetext{
${ }^{1}$ For better understanding, we keep the indexing of congruences by sets of variables throughout the proof of 3.12, even though Lemma 3.6 would allow us to drop them.
} 
Example 4.2. $\quad$ (i) The presentation of $\mathbb{P}_{\omega}$ in Example 3.8(iii) is reduced. For every term $\sigma_{n}\left(x_{0}, \ldots, x_{n-1}\right)$ let $k$ be the number of elements of $\left\{x_{i}\right\}_{i<n}$. Then there are variables $x_{i_{1}}, \ldots, x_{i_{k}}$ representing all $x_{0}, \ldots, x_{n-1}$. The term $\sigma_{k}\left(x_{i_{1}}, \ldots, x_{i_{k}}\right)$ is reduced and $E$ contains

$$
\sigma_{n}\left(x_{0}, \ldots, x_{n-1}\right)=\sigma_{k}\left(x_{i_{1}}, \ldots, x_{i_{k}}\right) .
$$

(ii) The presentations of the unordered-pairs functor in Example 3.8(i) is reduced, since every term is reduced.

(iii) Let $G$ be the quotient of $X^{\omega}$ modulo the congruence $\sim$ given by

$$
\left(x_{n}\right)_{n \in \omega} \sim\left(y_{n}\right)_{n \in \omega} \text { iff } x_{n}=y_{n} \text { for all but finitely many } n \in \omega \text {. }
$$

There is an obvious presentation of $G$ : use one $\omega$-ary operation and the equations between congruent terms. This presentation is not reduced. In fact, $G$ does not have any reduced presentation, see the theorem below.

(iv) A filter on a set $X$ is a collection of subsets $\mathcal{G} \subseteq \mathbb{P}(X)$ closed under finite intersections and supersets. The filter functor $\mathbb{F}$ associates to each set $X$ the set of all filters on $X$, and to each map $f: X \rightarrow Y$ the map $\mathbb{F} f: \mathbb{F} X \rightarrow \mathbb{F} Y$ which sends a filter $\mathcal{G}$ on $X$ to the filter $\mathbb{F} f(\mathcal{G}):=\{V \subseteq$ $\left.Y \mid f^{-1}[V] \in \mathcal{G}\right\}$. The filter functor weakly preserves pullbacks, but does not preserve wide intersections, see [4].

Theorem 4.3. A sound accessible set functor preserves wide intersections iff it has a reduced presentation.

Proof. In [5], Theorem 7.4(ii), it is shown that a sound set functor $F$ preserves wide intersections if and only if for every set $X$ and for each $a \in F X$ the filter

$$
\mu_{X}(a)=\left\{U \subseteq X \mid a \in F \iota_{U}^{X}[F U]\right\}
$$

is a principal filter. (Here $\iota_{U}^{X}: U \rightarrow X$ denotes subset inclusion.) That means:

for each $a \in F X$ there is a smallest $U \subseteq X$ with $a=F \iota_{U}^{X}(b)$ for some $b \in F U$.

If $F$ is accessible and $\varepsilon: H_{\Sigma} \rightarrow F$ is a surjective natural transformation, the elements of $F X$ are exactly the elements of the form $\varepsilon_{X}(\sigma(x))$ where $x: \operatorname{ar}(\sigma) \rightarrow X$. Therefore, the above condition translates into:

For each term $\sigma(x)$ with variables from $X$ there exists a smallest $U \subset X$ such that for some $\varepsilon_{U}(\tau(u))$ with $u: \operatorname{ar}(\tau) \rightarrow U$ we have

$$
\begin{aligned}
\varepsilon_{X}(\sigma(x)) & =F_{\iota_{U}}^{X}\left(\varepsilon_{U}(\tau(u))\right) \\
& =\varepsilon_{X}\left(H_{\Sigma} \iota_{U}^{X}(\tau(u))\right) \\
& =\varepsilon_{X}\left(\tau\left(\iota_{U}^{X} \circ u\right)\right) .
\end{aligned}
$$

In other words: For each $\sigma(x)$ with variables from $X$ there exists a smallest $U \subseteq X$ such that $E \vdash \sigma(x)=\tau(u)$ for some $\tau(u)$ with variables in $U$.

Example 4.4. The functor $G$ of Example 4.2(iii) does not have a reduced presentation, since it does not preserve intersections: consider e.g. the empty intersection of the subobjects

$$
\omega-k=\{k, k+1, k+2, \ldots\} \hookrightarrow \omega
$$

and observe that $G$ maps each of these subobjects to an isomorphism.

Proposition 4.5. A sound set functor preserves wide intersections iff it is a classdirected union of functors having reduced presentations. 
Proof. If $F$ is sound and preserves wide intersections, then each one of the functors $F_{\lambda}$ of $(3.2)$ is clearly sound. And $F_{\lambda}$ preserves wide intersections: given a wide pullback (2.4) and an element lying in $F_{\lambda} A$ and in the image of each $F_{\lambda} a_{i}$, then that element lies in $F B$. Since $F_{\lambda}$ is accessible, it has a reduced presentation.

Conversely, if $F=\bigcup_{i \in I} F_{i}$ is a class-directed union of functors $F_{i}$ with reduced presentations, then the fact that $F$ is sound implies (due to $F \emptyset=\bigcup_{i \in I} F_{i} \emptyset$ being $\lambda$-directed for for any infinite cardinal $\lambda>\operatorname{card}(F \emptyset))$ that there exists $i_{0} \in I$ with $F_{i}$ sound for every $i \geq i_{0}$.

Then each $F_{i}$, with $i \geq i_{0}$, preserves wide intersections, from which we conclude that $F$ does, too.

Remark 4.6. Let us call an element $a \in F A$ minimal, if no proper subobject $m$ : $A^{\prime} \hookrightarrow A$ has the property that $a$ lies in the image of $F m$. Observe that a sound set functor $F$ preserves wide intersections iff every element $a \in F A$ possesses a minimal subset, i.e. a subset $m: A^{\prime} \hookrightarrow A$ such that for some minimal element $a^{\prime} \in F A^{\prime}$ we have $a=F m\left(a^{\prime}\right)$.

\section{Weak preservation of Pullbacks}

In the present section we characterize set functors weakly preserving pullbacks. This is a condition which is often imposed on coalgebraic type functors, since it has numerous pleasant coalgebraic consequences. One of the most important ones being that observational equivalence and bisimilarity coincide.

\section{Example 5.1.}

(i) Given a filter $\mathcal{F}$ on the cardinal $n$, we have a congruence $\equiv_{\mathcal{F}}$ on $Q_{n}$ by defining for $u, v: n \rightarrow X$ :

$$
u \equiv_{\mathcal{F}} v \text { iff }\{x \in n \mid u(x)=v(x)\} \in \mathcal{F} .
$$

The corresponding functor

$$
Q_{n} / \equiv_{\mathcal{F}}
$$

is called filtered hom-functor. Every filtered hom-functor preserves pullbacks (strongly). Thus, also directed unions of filtered hom-functors preserve pullbacks. In fact, functors preserving pullbacks are characterized in [17](VIII.5 and VII.10) as the directed unions of filtered hom-functors.

(ii) For every group $S$ of bijections on the set $n$ ( a cardinal) we denote by

$$
Q_{n} / S
$$

the quotient of $Q_{n}$ (see 3.1) modulo the congruence $\sim_{S}$ which for $u, v$ : $n \rightarrow X$ is defined by

$$
u \sim_{S} v \text { iff } u=v \circ s \text { for some } s \in S .
$$

Each of these functors weakly preserves pullbacks (even wide pullbacks, see Section 6).

(iii) The finite powerset functor $\mathbb{P}_{\omega}$ weakly preserves pullbacks. In fact, given a pullback (2.1) and elements $a_{i} \in \mathbb{P}_{\omega} A_{i}$ with $\mathbb{P}_{\omega} f_{1}\left(a_{1}\right)=\mathbb{P}_{\omega} f_{2}\left(a_{2}\right)$ choose $x=p_{1}^{-1}\left(a_{1}\right) \times p_{2}^{-1}\left(a_{2}\right) \in \mathbb{P}_{\omega} P$ to get $a_{i}=\mathbb{P}_{\omega} p_{i}(x)$ for $i=1,2$.

We now turn to a characterization of functors weakly preserving (binary) pullbacks. Here, like in the case of wide intersections, we use a syntactic condition. 
Remark 5.2.

(i) The rule (3.7) implies that given an equation $\sigma\left(i d_{n}\right)=\rho(u)$ in a presentation $E$, then every flat term formed via $\sigma$ can be substituted by one formed by $\rho$ : for every $n$-tuple $x$ of variables, we have $E \vdash \sigma(x)=\rho(x \circ u)$. We can say that $\rho$ dominates $\sigma$.

(ii) Suppose that $\rho$ dominates two symbols $\sigma$ and $\tau$ : $E \vdash \sigma\left(i d_{n}\right)=\rho(u)$ and $E \vdash \tau\left(i d_{m}\right)=\rho(v)$. Then we clearly derive $E \vdash \sigma(x)=\tau(y)$ whenever $x \circ u=y \circ v$. We call such equations $\sigma(x)=\tau(y)$ consequences of the joint domination by $\rho$.

Definition 5.3. A flat equational presentation $(\Sigma, E)$ is called dominated provided that every equation derivable from $E$ is a consequence of some joint domination.

Explicitly, an equational presentations $(\Sigma, E)$ being dominated means, that for every equation $\sigma(x)=\tau(y)$ derivable from $E$ (where $\sigma \in \Sigma_{n}, x: n \rightarrow X, \tau \in \Sigma_{m}, y$ : $m \rightarrow X)$ there exists an operation symbol $\rho$ in $\Sigma_{k}$ and two $k$-tuples $u \in n^{k}$ and $v \in m^{k}$ such that

$$
\begin{aligned}
& E \vdash \quad \rho(u)=\sigma\left(i d_{n}\right) \\
& E \vdash \quad \rho(v)=\tau\left(i d_{m}\right)
\end{aligned}
$$

and

$$
x \circ u=y \circ v: k \rightarrow X .
$$

Example 5.4. (i) The equational presentation of $\mathbb{P}_{\omega}$ in Example 3.8 is dominated: given $\sigma_{n}(x)=\sigma_{m}(y)$ in $E$ where $\left\{x_{i}\right\}=\left\{y_{j}\right\}$ is a set of $k$ elements, then let $u: k \rightarrow n$ and $v: k \rightarrow m$ be such that $x \circ u=y \circ v$. The equations $\sigma_{n}\left(i d_{n}\right)=\sigma_{k}(u)$ and $\sigma_{m}\left(i d_{m}\right)=\sigma_{k}(v)$ lie in $E$.

(ii) The functor $Q_{n} / \equiv_{\mathcal{F}}$ above has a dominated presentation by a single $n$-ary equation symbol $\sigma$ and by the equations

$$
\sigma\left(i d_{n}\right)=\sigma(w)
$$

where $w$ ranges over all endofunctions of $n$ whose set of fixed points lies in $\mathcal{F}$. Here we choose, of course, $\rho=\sigma, u=w$ and $v=i d$.

Theorem 5.5. A sound accessible functor weakly preserves pullbacks if it has a dominated presentation.

Proof. Let $F$ be a sound functor with a dominated presentation $(\Sigma, E)$ and let

$$
\varepsilon: H_{\Sigma} \rightarrow F
$$

be the corresponding natural transformation. Given a pullback

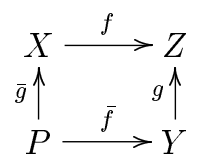

and elements $x \in F X, y \in F Y$ with $F f(x)=F g(y)$, we are to find $z \in F P$ with $x=F \bar{g}(z)$ and $y=F \bar{f}(z)$. Choose $\sigma\left(x_{i}\right) \in H_{\Sigma} X$ and $\tau\left(y_{j}\right) \in H_{\Sigma} Y$ with 


$$
\begin{aligned}
& x=\varepsilon_{X}\left(\sigma\left(x_{i}\right)\right) \text { and } y=\varepsilon_{Y}\left(\tau\left(y_{j}\right)\right) \text {. Then } \varepsilon_{Z} \text { merges } \sigma\left(f\left(x_{i}\right)\right) \text { and } \tau\left(g\left(y_{j}\right)\right): \\
& \varepsilon_{Z}\left(\sigma\left(f\left(x_{i}\right)\right)\right.=\varepsilon_{Z} \circ F_{\Sigma} f\left(\sigma\left(x_{i}\right)\right) \\
&=F f \circ \varepsilon_{X}\left(\sigma\left(x_{i}\right)\right) \\
&=F f(x) \\
&=F g(y) \\
&=\varepsilon_{Z}\left(\tau\left(g\left(y_{j}\right)\right) .\right.
\end{aligned}
$$

Consequently,

$$
E \vdash \sigma(f \circ x)=\tau(g \circ y) .
$$

Then we have, by the definition of dominated presentation, equations $E \vdash \sigma\left(i d_{n}\right)=$ $\rho(u)$ and $E \vdash \tau\left(i d_{m}\right)=\rho(v)$ such that

$$
f \circ x \circ u=g \circ y \circ v \text {. }
$$

Choose $\bar{z}$ such that the diagram

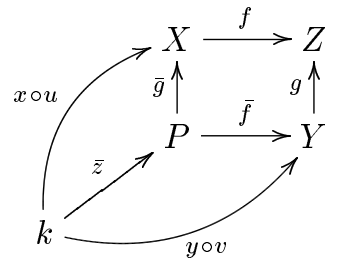

commutes. Then the element

$$
z=\varepsilon_{P}(\rho(\bar{z}))
$$

fulfills

$$
\begin{aligned}
F \bar{g}(z) & =\varepsilon_{X} \circ H_{\Sigma} \bar{g}(\rho(\bar{z})) \\
& =\varepsilon_{X}(\rho(\bar{g} \circ \bar{z})) \\
& =\varepsilon_{X}(\rho(x \circ u)) \\
& =\varepsilon_{X} \circ H_{\Sigma} x(\rho(u)) \\
& =F x \circ \varepsilon_{n}\left(\sigma\left(i d_{n}\right)\right) \\
& =\varepsilon_{X} \circ H_{\Sigma} x\left(\sigma\left(i d_{n}\right)\right) \\
& =\varepsilon_{X}(\sigma(x)) \\
& =x
\end{aligned}
$$

and analogously $F \bar{f}(z)=y$.

Conversely, if $F$ is a sound $\lambda$-accessible functor weakly preserving pullbacks, we prove that the presentation of Proposition 3.9 is dominated. Given an equation $\sigma(x)=\tau(y)$ in $E$ :

$$
F x(\sigma)=F y(\tau)
$$

for $x: n \rightarrow Z, y: m \rightarrow Z$, we form the pullback

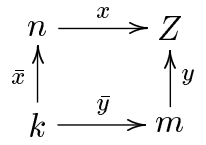

and assume, without loss of generality, that the domain $k$ is a cardinal. Since $n<\lambda$ and $m<\lambda$, we conclude from Formula (2.3) that $k<\lambda$. Moreover, there exists 
$\rho \in F(k)$ such that $F \bar{x}(\rho)=\sigma$ and $F \bar{y}(\rho)=\tau$ and this is a $k$-ary operation symbol such that $E$ contains $\sigma\left(i d_{n}\right)=\rho(\bar{x})$ and $\tau\left(i d_{n}\right)=\rho(\bar{y})$. Put $u=\bar{x}$ and $v=\bar{y}$.

Since directed unions commute with pullbacks in Set, we obtain from Remark 3.10 immediately:

Corollary 5.6. A sound functor weakly preserves pullbacks iff it is a directed union of functors having dominated presentations.

\section{Weak Preservation of Wide Pullbacks}

Finitary set functors weakly preserving wide pullbacks were characterized by A. Joyal [10] as precisely the analytic functors. We generalize the concept of analytic functor from finitary to accessible, and prove that these functors are precisely those weakly preserving wide pullbacks. Our proof follows the ideas of R. Hasegawa [9].

Notation 6.1. For a cardinal $\lambda$ we denote by $\mathcal{B}_{\lambda}$ the category of all sets of cardinalities less than $\lambda$ and all bijections.

Definition 6.2. A set functor $F$ is called analytic if it is, for some cardinal $\lambda$, the left Kan extension of a functor from $\mathcal{B}_{\lambda}$ to Set. Equivalently: $F$ is a coproduct of the functors $Q_{n} / S$ of Example 5.1(ii) where $S$ is a group of permutations on a cardinal $n<\lambda$.

\section{Example 6.3.}

(i) Polynomial functors are analytic. They preserve wide pullbacks (strongly). In fact, these are the only set functors preserving wide pullbacks, see [14]. Indeed, if $F$ preserves wide pullbacks and $F=\bigsqcup_{i \in I} F_{i}$ with $F_{i} 1 \cong 1$, see Formula (2.5), then each $F_{i}$ preserves wide pullbacks, thus it preserves limits. By the Special Adjoint Functor Theorem, each $F_{i}$ is representable. Thus, $F$ is polynomial.

(ii) The powerset functor $\mathbb{P}$ weakly preserves wide pullbacks, the argument is analogous to Example 5.1(iii). None of the subfunctors $\mathbb{P}_{n}$ (of all subsets of cardinalities less than $n$ ) with $n>3$ is analytic - and none of them weakly preserves wide pullbacks, as the next theorem demonstrates.

(iii) The functor

$$
e^{X}=\coprod_{n \in \omega} X^{n} / n !
$$

where $n$ ! denotes the symmetric group (of all permutations) on $n$ is analytic.

Remark 6.4. Before characterizing functors weakly preserving wide pullbacks, let us compare this with weak preservation of products. Although a product $A=\Pi_{i \in I} X_{i}$ with projections $\pi_{i}$ is a wide pullback of the trivial cocone $\left(!_{X_{i}}: X_{i} \rightarrow 1\right)_{i \in I}$

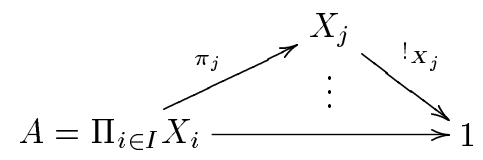

it is not true that a functor $F$ weakly preserving pullbacks must also weakly preserve products. The powerset functor $\mathbb{P}$, for example, does not weakly preserve the product $\emptyset \times 1$. However, the implication does hold whenever $F$ is indecomposable, i.e. $F 1 \cong 1$. Observe that weak preservation of products states precisely that 
given a set of elements $a_{i} \in F A_{i}(i \in I, I$ any set $)$ then there exists an element $a \in F \prod_{i \in I} A_{i}$ such that for all $i \in I$ we have $a_{i}=F \pi_{i}(a)$. We thus derive the following

Lemma 6.5. An accessible functor weakly preserves products iff it is a quotient of a hom-functor.

Proof. Let $F$ be accessible and weakly preserve products. Since $F$ is accessible, there is a set $Y$ such that for every set $X$ and every $x \in F X$ there is a map $f: Y \rightarrow X$ and some $y \in F Y$ such that $(F f)(y)=x$. Choose $A$ to be the $Y$-th power of $Y$. Since $F$ weakly preserves products, there exists $a \in F A$ such that

$$
F Y=\{F f(a) \mid f: A \rightarrow Y\} .
$$

Then $F$ is a quotient of $\operatorname{hom}(A,-)$ because the Yoneda transformation $f \mapsto F f(a)$ is an epimorphism $\varepsilon: \operatorname{hom}(A,-) \rightarrow F$.

Conversely, every quotient $\operatorname{hom}(A,-) / \sim$ weakly preserves products: given elements $x_{i}=\left[f_{i}\right]$ of $\operatorname{hom}\left(A, X_{i}\right) / \sim_{X_{i}}$ for $f_{i}: A \rightarrow X_{i}$, then $f=\left\langle f_{i}\right\rangle: A \rightarrow \Pi X_{i}$ fulfils

for every $i \in I$.

$$
F \pi_{i}([f])=\left[p_{i} \circ f\right]=\left[f_{i}\right]=x_{i}
$$

Theorem 6.6. An accessible set functor weakly preserves wide pullbacks iff it is analytic.

Proof. (1) Let $F$ be $\lambda$-accessible and weakly preserve wide pullbacks. It is sufficient to prove that

$$
F \text { indecomposable } \Longrightarrow F \cong Q_{n} / S
$$

for some group $S$ of permutations on $n$. In fact, the general case follows since every component, see (2.5), of a functor weakly preserving wide intersections also has this property.

Since $F$ is accessible and preserves weak products, there is a set $A$ and an element $a \in F A$ such that for every set $X$

$$
F X=\{F f(a) \mid f: A \rightarrow X\} .
$$

Moreover, since $F$ preserves intersections, we can choose $A$ to be minimal, see Remark 4.6. Consequently, given a morphism $f: B \rightarrow A$ then

$$
a \in F f[F B] \text { implies that } f \text { is an epimorphism. }
$$

We call the element $a \in F A$ analytic, provided that it has the property that given a parallel pair $u, v: A \rightarrow X$ and $F u(a)=F v(a)$ then $u=v \circ s$ for some bijection $s: A \rightarrow A$ with $F s(a)=a$. If we prove that $F$ has an analytic element, we are ready: let $S$ be the group of all bijections $s: A \rightarrow A$ with $F s(a)=a$, then we claim that $F$ is the quotient

$$
F \cong \operatorname{hom}(A,-) / \sim
$$

where $u \sim v$ iff $u=v \circ s$ for $s \in S$.

In fact, the Yoneda transformation $\operatorname{hom}(A,-) \rightarrow F$ of $a \in F A$ factorizes through $\sim$ via a natural isomorphism; this follows from $a$ being analytic.

Assuming no analytic element exists, we derive a contradiction. We will define a transfinite chain of objects $A_{i}$, elements $a_{i} \in F A_{i}$ and connecting maps

$$
e_{j i}: A_{j} \rightarrow A_{i}
$$


for $(i \leq j)$ with $F e_{j i}\left(a_{j}\right)=a_{i}$ such that for $i<j$ each $e_{j i}$ is an epimorphism but not an isomorphism, and $A_{i}$ is minimal for $a_{i}$.

This is the desired contradiction: since $F$ is $\lambda$-accessible, we have by Formula (3.1) that minimal sets of elements of $F$ cannot have cardinalities beyond $\lambda$. However, since $e_{j i}$ is not an isomorphism, for all $i<j$, we see that $A_{i}$ has cardinality at least that of $i$ for every ordinal $i$.

Initial step: $\left(A_{0}, a_{0}\right)=(A, a)$ above.

Limit step: Given a limit ordinal $j$, let

$$
\hat{e}_{j i}: \hat{A}_{j} \rightarrow A_{i}
$$

for $i<j$ be a limit of the preceding $j$-chain. Then each $\hat{e}_{j i}$ is an epimorphism, see (6.3), but not an isomorphism (since the preceding chain had that property). Moreover, the limit cone is also the wide pullback of the cocone of all $e_{i, 0}: A_{i} \rightarrow A$ for $i<j$. Since $F$ weakly preserves this wide pullback, there exists $\hat{a}_{j} \in F \hat{A}_{j}$ with $F \hat{e}_{j i}\left(\hat{a}_{j}\right)=a_{i}$ for all $i<j$. Let $m: A_{j} \hookrightarrow \hat{A}_{j}$ be a minimal set for the element $\hat{a}_{j}$ and let $a_{j} \in F A_{j}$ fulfill $\hat{a}_{j}=F m\left(a_{j}\right)$. Denote by

$$
e_{j i}=\hat{e}_{j i} \circ m: A_{j} \rightarrow A_{i}
$$

for $i<j$ the domain restriction of the above cone. Then we have

$$
F e_{j i}\left(a_{j}\right)=a_{j}
$$

for $i<j$ and this implies that $e_{j i}$ is an epimorphism.

Isolated step: Given $\left(A_{i}, a_{i}\right)$ then since $a_{i}$ is not analytic, we have a pair $u, v$ : $A \rightarrow X$ with $F u\left(a_{i}\right)=F v\left(a_{i}\right)$ and $u \neq v \circ s$ for all bijections $s: A \rightarrow A$ with $F s\left(a_{i}\right)=a_{i}$. Since $F$ weakly preserves the pullback of $u$ and $v$,

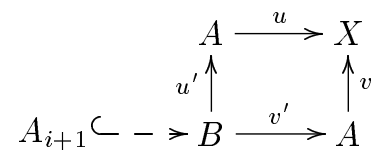

there exists $b \in F B$ with $F u^{\prime}(b)=F v^{\prime}(b)=a_{i}$. Let $m: A_{i+1} \hookrightarrow B$ be a minimal set for $b$ and let $a_{i+1}$ be an element of $F A_{i+1}$ with $b=F m\left(a_{i+1}\right)$. From

$$
F\left(u^{\prime} \circ m\right)\left(a_{i+1}\right)=a_{i}
$$

we conclude that $u^{\prime} \circ m$ is an epimorphism. If this is not an isomorphism, put

$$
e_{i+1, i}=u^{\prime} \circ m \text {. }
$$

If $u^{\prime} \circ m$ is an isomorphism but $v^{\prime} \circ m$ is not, put

$$
e_{i+1, i}=v^{\prime} \circ m \text {. }
$$

Observe that it is not possible that both $u^{\prime} \circ m$ and $v^{\prime} \circ m$ are isomorphisms, for then $s=\left(v^{\prime} \circ m\right) \circ\left(u^{\prime} \circ m\right)^{-1}$ would be an isomorphism with $F s\left(a_{i}\right)=a_{i}$ and

$$
v \circ s=v \circ v^{\prime} \circ m \circ\left(u^{\prime} \circ m\right)^{-1}=u \circ\left(u^{\prime} \circ m\right) \circ\left(u^{\prime} \circ m\right)^{-1}=u \text {. }
$$

(2) The converse implication

analytic $\Longrightarrow$ weakly preserves wide pullbacks 
is easy. Since coproducts commute with wide pullbacks in Set, it is sufficient to verify that each $Q_{n} / S$ weakly preserves wide pullbacks. Let

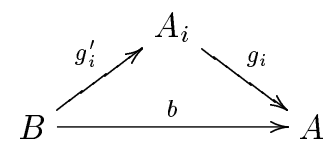

be a wide pullback and let $\left[x_{i}\right] \in A_{i}^{n} / S$ be classes of $n$-tuples with

$$
Q_{n} / S\left(g_{i}\right)\left[x_{i}\right]=[y]
$$

independent of $i$. Then $y: n \rightarrow A$ satisfies: for every $i \in I$ there is $s_{i} \in S$ with

$$
y=g_{i} \circ x_{i} \circ s_{i} \text {. }
$$

Consequently, there exists $z: n \rightarrow B$ with

$$
g_{i}^{\prime} \circ z=x_{i} \circ s_{i}
$$

which means that

$$
Q_{n} g_{i}^{\prime}(z) \sim_{S} x_{i}
$$

thus

for $i \in I$.

$$
Q_{n} / S\left(g_{i}^{\prime}\right)[z]=\left[x_{i}\right]
$$

Remark 6.7. The characterization in Theorem 5.5 is of course much less intuitive than Joyal's result for wide pullbacks. This can be mended for the functors called super-finitary in [2]: these are precisely the quotient functors of $H_{\Sigma}$ where $\Sigma$ is a finite and finitary signature. The proof of the following theorem presented in [2] was very complicated. But it is easy to see how the technique of Theorem 6.6 above immediately adapts to the proof of

Theorem 6.8. A super-finitary functor weakly preserves pullbacks iff it is analytic.

Remark 6.9. Unfortunately, there does not seem to be a "nice" characterization of set functors (not necessarily accessible) that weakly preserve wide pullbacks. Observe e.g. that although $\mathbb{P}$ has this property, none of its proper subfunctors not contained in $\mathbb{P}_{3}$ shares that property. In fact, it is easy to verify that $\mathbb{P}$ has no proper subfunctors besides $\mathbb{P}_{n}$ for cardinals $n$. Thus class-directed unions of accessible subfunctors do not help. In contrast, we have:

Theorem 6.10. A sound functor weakly preserves products iff it is a class-directed union of quotients of hom-functors.

Proof. Let $F$ weakly preserve products. For each cardinal $n$ there exists, by Remark 6.4 , an element $a_{n}$ in the $n$-th power of $n$, such that the subfunctor $F^{[n]}$ generated by $a_{n}$

$$
F^{[n]} X=\left\{F f\left(a_{n}\right) \mid f: A_{n} \rightarrow X\right\}
$$

contains the $n$-accessible subfunctor $F_{n}$ of Formula (3.2). Since $F^{[n]}$ is generated by a single element, it clearly shares with $F$ the property of weakly preserving products. Thus it is a quotient of a hom-functor, by Lemma 6.5. From $F_{n} \subseteq F^{[n]}$ we get

$$
F=\bigcup_{n \in \text { Card }} F^{[n]} .
$$

The converse implication is trivial. 


\section{Preservation of Inverse Images}

As we have remarked earlier, a functor preserves weak pullbacks iff it weakly preserves inverse images and kernel pairs. Observe that a sound functor preserves inverse images iff it preserves them weakly.

Preservation of inverse images plays a central role in the theory of recursive coalgebras, see [15]. Also, as has been argued in [5], functors preserving inverse images can be considered as generalized container types.

Indeed, given any functor $F$, then the "generalized elements" of any container $c \in F(X)$ can be defined as

$$
\mu_{X}(c):=\left\{U \subseteq X \mid c \in F \iota_{U}^{X}[F U]\right\},
$$

where $\iota_{U}^{X}: U \hookrightarrow X$ denotes subset inclusion. (In a different notation, the filters $\mu_{X}(c)$ were already introduced and used by V. Koubek [11]). Due to Proposition 2.2, $\mu$ is a transformation from $F$ to the filter functor $\mathbb{F}$, see Example 5.1. For a general functor, $\mu$ need not be natural, but it is always sub-natural, meaning that the naturality square commutes whenever $f: X \rightarrow Y$ is monic:

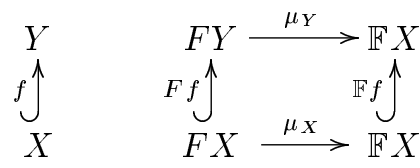

Moreover, $\mu$ is sub-cartesian, meaning that the naturality squares are pullback squares for monic $f: X \rightarrow Y$. In fact, it was shown in [5] that $F$ preserves inverse images iff $\mu$ is a natural transformation. A related characterization from [5] is:

Proposition 7.1. A set functor preserves inverse images if and only if there exists a natural and sub-cartesian transformation to the filter functor.

Combining this with Proposition 2.7 and using the fact that by Lemma 2.1 the composition of two sub-cartesian transformations is sub-cartesian, we infer a result first proved using coalgebraic arguments by T. Schröder in his thesis [14]:

Proposition 7.2. If a sound functor preserves inverse images, then so do all sound subfunctors.

For finitary functors, see section 3.1, preservation of inverse images has also been characterized in [1]. Recall that in general algebra an equation is called regular if it has the same variables on both sides. The sound finitary functors preserving inverse images are precisely those having an equational presentations by regular equations.

Example 7.3. $\quad$ (i) Every polynomial functor $H_{\Sigma}$ preserves inverse images.

(ii) The class of inverse image preserving functors is closed under products and coproducts.

(iii) The functors $\mathbb{P}, \mathbb{P}_{\omega}$ preserve inverse images.

(iv) The functor $D$ of Example 3.3(ii) does not preserve inverse images, neither does its sound modification $D^{\prime}$ with $D^{\prime} \emptyset=1$. In fact, consider the inverse image of the embedding $1 \hookrightarrow 2$ (explicitly: $\{0\} \hookrightarrow\{0,1\}$ ) under 
the constant map $c_{1}: 2 \rightarrow 2$ with value $1:$

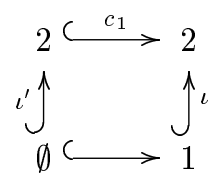

For the elements $(0,1) \in D 2$ and $d \in D 1$ with

$$
D c_{1}(0,1)=d=D \iota(d)
$$

there is no element of $D^{\prime} \emptyset$ which $D^{\prime} \iota^{\prime}$ would map to $(0,1)$.

(v) The functor $G$, see Example 4.2(iii), preserves inverse images, as we shall derive from the characterization theorem below. But it clearly has no presentations by regular equations. We thus need a new idea for infinitary signatures.

Definition 7.4. Let $(\Sigma, E)$ be an equational presentation. We say that a term $\sigma\left(x_{i}\right)_{i<n}$ depends only on a subset $a: A \hookrightarrow n$ provided that for every $m$-tuple $y$ of variables with $x \circ a=y \circ a$ we have

$$
E \vdash \sigma\left(x_{i}\right)_{i<n}=\sigma\left(y_{j}\right)_{j<m} .
$$

The presentation is called almost regular if for every equation $E \vdash \sigma\left(x_{i}\right)_{i<n}=$ $\tau\left(y_{j}\right)_{j<m}$ the term $\sigma\left(x_{i}\right)$ only depends on the variables that appear on both sides. (That is, $\sigma\left(x_{i}\right)$ only depends on $\left\{i \mid x_{i}=y_{j}\right.$ for some $\left.j\right\}$.)

Theorem 7.5. A sound accessible functor preserves inverse images iff it has an almost regular presentation.

Proof. (1) Let $(\Sigma, E)$ be an almost regular presentation of a sound functor $F$, and let

$$
\varepsilon: H_{\Sigma} \rightarrow F
$$

be the corresponding transformation. Given an inverse image

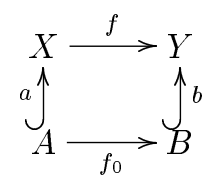

we are to find, for any pair $p \in F X, q \in F B$ with

$$
F f(p)=F b(q)
$$

an element $r \in F A$ with

$$
F a(r)=p
$$

Since $F$, being sound, preserves monomorphisms, it then follows that $F f_{0}(r)=q$.

We can express

$$
p=\varepsilon_{X}(\sigma(x)) \text { and } q=\varepsilon_{B}(\tau(y))
$$


for some $\sigma \in \Sigma_{n}, x: n \rightarrow X$ and $\tau \in \Sigma_{m}, y: m \rightarrow B$. The naturality of $\varepsilon$ implies

$$
\begin{aligned}
\varepsilon_{Y}(\sigma(f \circ x)) & =\varepsilon_{Y} \circ H_{\Sigma} f(\sigma(x)) \\
& =F f \circ \varepsilon_{X}(\sigma(x)) \\
& =F f(p) \\
& =F b(q) \\
& =\varepsilon_{Y}(\tau(b \circ y)) .
\end{aligned}
$$

Consequently,

$$
E \vdash \sigma(f \circ x)=\tau(b \circ y)
$$

and we conclude that $\sigma(f \circ x)$ only depends on

$$
\left\{i<n \mid f\left(x_{i}\right) \in B\right\}=\left\{i<n \mid x_{i} \in A\right\} .
$$

Choose $x^{\prime}: n \rightarrow A$ with $x_{i}^{\prime}=x_{i}$ whenever $x_{i} \in A$, then $E \vdash \sigma(x)=\sigma\left(x^{\prime}\right)$ and $x=a \circ x^{\prime}$. The desired element $r$ with $F a(r)=p$ is $r=\varepsilon_{A}\left(\sigma\left(x^{\prime}\right)\right)$ :

$$
F a(r)=F a \circ \varepsilon_{A}\left(\sigma\left(x^{\prime}\right)\right)=\varepsilon_{X}\left(\sigma\left(a \circ x^{\prime}\right)\right)=\varepsilon(\sigma(x))=p .
$$

(2) Let $F$ be accessible and preserve inverse images. Then we prove that the presentation of Proposition 3.9 is almost regular. Let

$$
E \vdash \sigma(x)=\tau(y)
$$

that is, let $x: n \rightarrow X, \sigma \in F n, y: m \rightarrow X$ and $\tau \in F m$ fulfill

$$
F x(\sigma)=F y(\tau) .
$$

Factorize $y$ as an epimorphism $y_{0}: m \rightarrow \bar{m}$ followed by a monomorphism $\bar{y}: \bar{m} \hookrightarrow$ $X$. It is our task to prove that $\sigma(x)$ only depends on the subset $p: k \hookrightarrow n$ in the inverse image

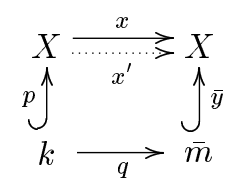

In fact, let $x^{\prime}: n \rightarrow X$ fulfill

$$
x^{\prime} \circ p=x \circ p \text {. }
$$

Since $F$ preserves the above pullback and $F x(\sigma)=F \bar{y}\left(F y_{0}(\tau)\right)$, there exists $\rho \in F k$ with $F p(\rho)=\sigma$. Then the desired conclusion

$$
E \vdash \sigma(x)=\sigma\left(x^{\prime}\right)
$$

follows from

$$
F x(\sigma)=F(x \circ p)(\rho)=F\left(x^{\prime} \circ p\right)(\rho)=F x^{\prime}(\sigma) .
$$

Corollary 7.6. A sound functor preserves inverse images iff it is a directed union of functors having almost regular presentations. 


\section{Conlusions and Open Problems}

We have characterized set functors having some of the properties frequently utilized in General Coalgebra. The most important of these is weak preservation of (binary) pullbacks: we characterize accessible functors that weakly preserve pullbacks as those that have a dominating equational presentation. The canonical presentation of the finite powerset functor is a prototypical example of such a dominating presentation. Unfortunately, we have no direct description of the weak preservation of pullbacks, and also no characterization that would be applicable to non-accessible functors.

The situation is better for functors weakly preserving wide pullbacks: André Joyal described all finitary functors with this property as being the analytic functors, that is, coproducts of representables modulo a group of isomorphisms. We prove the same result for accessible functors. However, also here we have no characterization for non-accessible functors. Why, for that matter, does the powerset functor weakly preserve wide pullbacks?

We further characterize the functors preserving (wide) intersections and inverse images. In both cases the characterization of accessible functors with that property is in terms of equational presentations, whereas general functors have that property iff they are class-directed unions of accessible functors with the same property.

\section{REFERENCES}

[1] J. Adámek, D. Lücke, and S. Milius. Recursive coalgebras of finitary functors. Theoretical Informatics and Applications, 41:447-463, 2007.

[2] J. Adámek and V. Trnková. Automata and Algebras in Categories. Kluwer Academic Publishers, 1990

[3] J. Adámek and H. Porst. From varieties of algebras to covarieties of coalgebras. In A. Corradini, M. Lenisa, and U. Montanari, editors, Coalgebraic Methods in Computer Science (CMCS'01), number 44.1 in Electronic Notes in Theoretical Computer Science, pages 27-46. Elsevier, 2001.

[4] H.P. Gumm. Functors for coalgebras. Algebra Universalis, 45:135-147, 2001.

[5] H.P. Gumm. From T-coalgebras to filter structures and transition systems. In D.H. Fiadeiro et al., editors, Algebra and Coalgebra in Computer Science, volume 3629 of Lecture Notes in Computer Science, pages 194-212. Springer, 2005.

[6] H.P. Gumm, J. Hughes, and T. Schröder. Distributivity of categories of coalgebras. Theoretical Computer Science, 308:131-143, 2003.

[7] H.P. Gumm and T. Schröder. Coalgebras of bounded type. Math. Struct. in Comp. Science, $12: 565-578,2002$.

[8] H.P. Gumm and T. Schröder. Types and coalgebraic structure. Algebra Universalis, 53:229$252,2005$.

[9] R. Hasegawa. Two applications of analytic functors. Theoretical Computer Science, $(272): 113-175,2002$

[10] A. Joyal. Foncteurs analytiques et espèces de structures. In B. Freitag et al., editors, Combinatoire énumérative, pages 126-159. Springer-Verlag, 1986.

[11] V. Koubek. Set functors. Comm. Math. Univ. Carolinae, 12(1):175-195, 1971.

[12] E.G. Manes. Implementing collection classes with monads. Math. Struct. in Comp. Science, 8:231-276, 1998

[13] J.J.M.M. Rutten. Universal coalgebra: a theory of systems. Theoretical Computer Science, $249(1): 3-80,2000$

[14] T. Schröder. Coalgebren und Funktoren. PhD thesis, FB Mathematik und Informatik, Philipps-Universität Marburg, 2001. http://archiv.ub.uni-marburg.de/diss/z2001/0205/.

[15] P. Taylor. Practical Foundations of Mathematics. Cambridge University Press, 1999.

[16] V. Trnková. Some properties of set functors. Comm. Math. Univ. Carolinae, (10,2):323-352, 1969. 
[17] V. Trnková. On descriptive classification of set-functors I. Comm. Math. Univ. Carolinae, 12(1):323-352, 1971

[18] V. Trnková. On descriptive classification of set-functors II. Comm. Math. Univ. Carolinae, 12(2):345-357, 1971 